\title{
Euzetrema knoepffleri n. gen., n. sp. (Monogenea), parasite interne d'un Amphibien endémique de Corse
}

\author{
Par Claude COMBES
}

Euproctus montanus (Savi 1838) est un Amphibien Urodèle, endémique de l'île de Corse, qui habite principalement les ruisseaux à eaux claires des montagnes, mais se rencontre également (Goux, 1953 et 1955) à des altitudes plus faibles et même dans des eaux légèrement saumâtres.

Les Euproctes que nous avons examinés proviennent de deux stations différentes: en mai 1963, M. Louis-Philippe Knoepffler, chargé de recherches au C.N.R.S., nous a adressé 22 animaux provenant de la forêt d'Aïtone et, en mai 1964, nous avons récolté nous-même 10 individus dans la forêt de l'Hospédale, au col d'Illara. Certains des Euproctes de ces deux stations présentaient, outre des Trématodes et Acanthocéphales intestinaux, des Monogènes parasites de la vessie urinaire. Nous avons été amené à créer, pour ces derniers, une espèce nouvelle appartenant à un genre nouveau. Nous en donnons la description ci-après.

\section{EUZETREMA KNOEPFFLERI n. gen., n. sp.}

Hôte: Euproctus montanus (Savi 1838).

Habitat: vessie urinaire.

Localités: forêts d'Aïtone et de l'Hospédale (Corse).

Matériel de description: neuf individus adultes.

\section{Description}

L'animal, extrêmement déformable sur le vivant, présente un corps plus ou moins aplati, fortement effilé vers l'avant, et prolongé dans sa région postérieure par un hapteur à concavité ventrale (fig. 1). La forme générale (excepté toutefois la structure du hapteur) rappelle de manière frappante celle des Polystomes de vessie, parasites habituels des Amphibiens Anoures. La longueur totale de l'animal varie de 1,70 à 
$1,90 \mathrm{~mm}$, sa largeur de 0,75 à $1,15 \mathrm{~mm}$; son épaisseur (mesurée sur coupes sériées) est voisine de $0,35 \mathrm{~mm}$. A l'extrémité antérieure, l'animal possède deux petites ventouses circulaires.

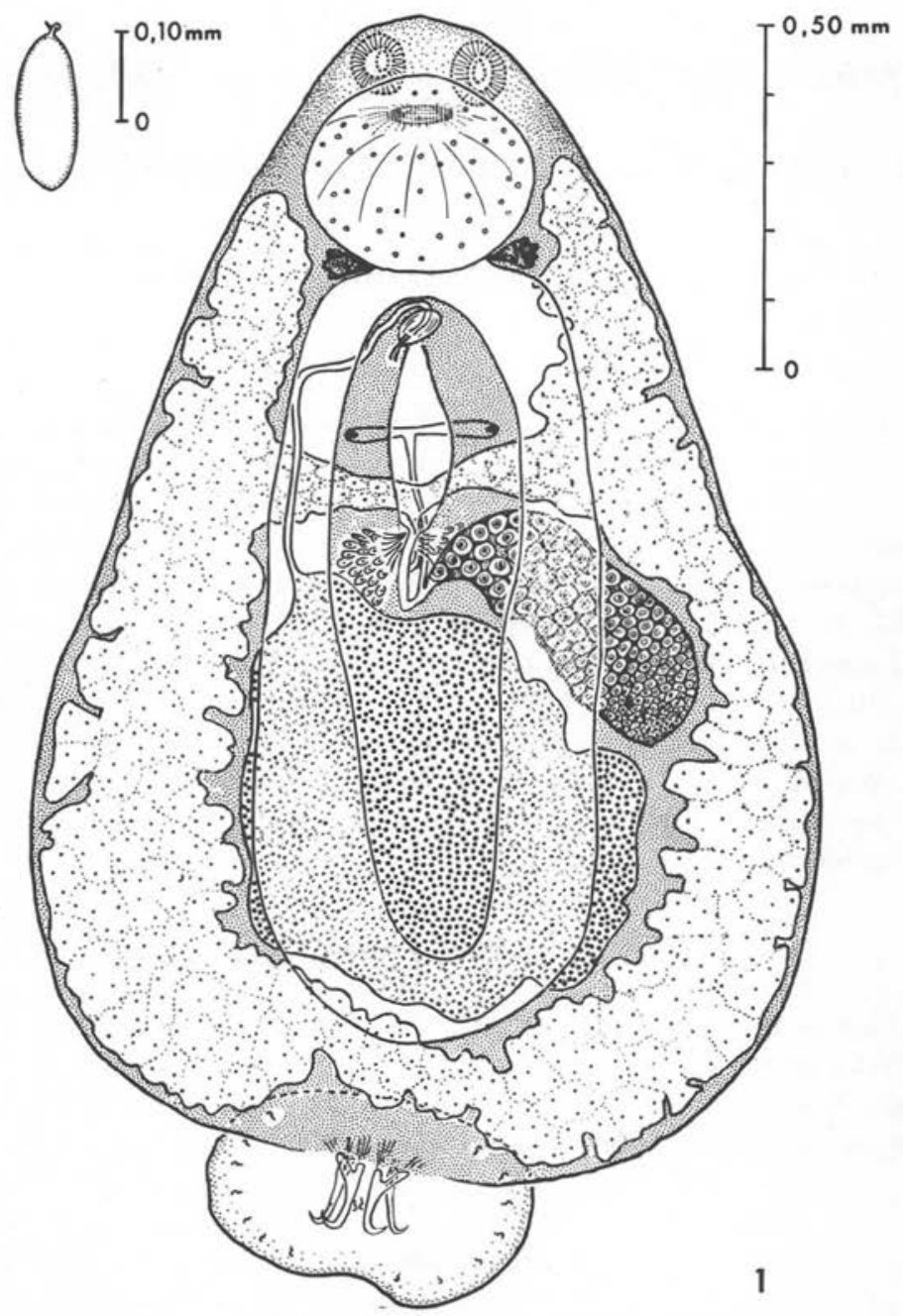

Fig. 1. - Euzetrema knoepffieri n. gen. n. sp. Vue dorsale.

VENTOUSES ANTÉRIEURES :

Elles sont antéro-latérales, petites, mais très déformables, visibles principalement sur le vivant. Elles sont indépendantes de l'appareil digestif et s'ouvrent sur la face ventrale; elles sont dépourvues de papilles. 


\section{HAPTEUR :}

Bien que parfaitement fonctionnel (nous avons observé le parasite en place dans la vessie de son hôte), il est de taille réduite (longueur : $0,30 \mathrm{~mm}$; largeur : $0,50 \mathrm{~mm}$ ). Il porte deux paires de grands crochets, des pièces intermédiaires et seize crochets larvaires.

a) Grands crochets (fig. 2): Les quatre crochets sont insérés à la face ventrale du hapteur. Nous distinguerons la paire de crochets la plus interne, c'est-à-dire la plus proche du plan de symétrie de l'animal, et la paire la plus externe.

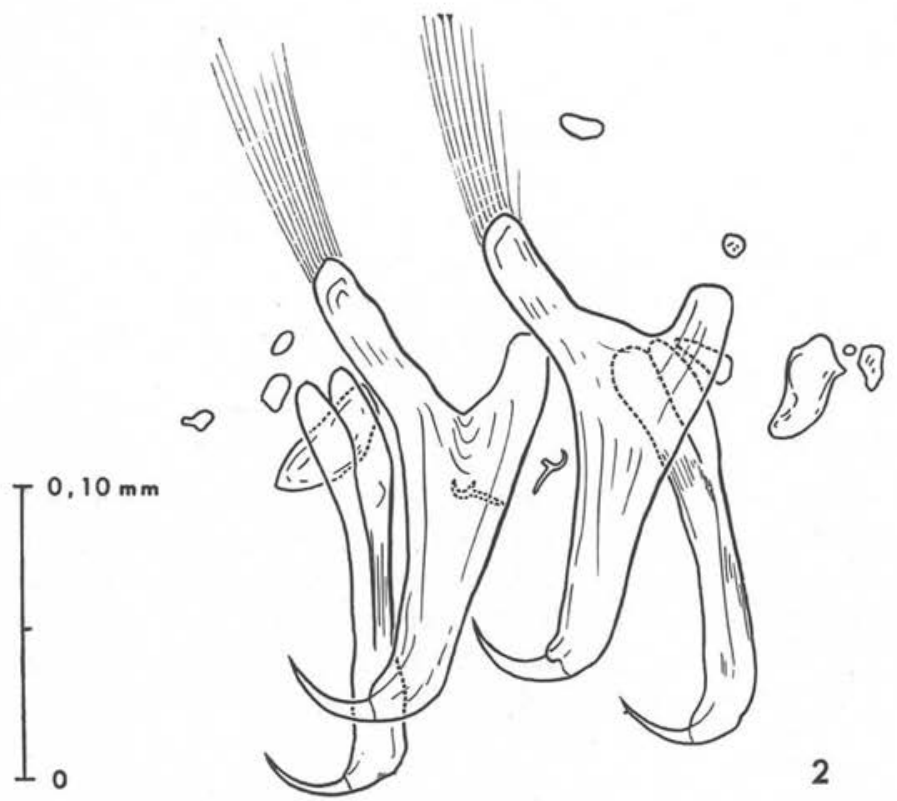

Fig. 2. - E. knoepffleri : détail des grands crochets du hapteur.

Les crochets de la paire la plus interne présentent un manche sub-rectiligne sur lequel s'insère obliquement une garde puissante ; le manche est terminé par une forte pointe recourbée. Le manche mesure $0,142 \mathrm{~mm}$ de longueur et, à son extrémité antérieure (c'est-à-dire à l'opposé de la pointe), $0,015 \mathrm{~mm}$ de largeur. La garde fait avec le manche un angle de $45^{\circ}$ et s'élargit fortement au niveau de son insertion; elle est de manière constante légèrement plus longue que le manche ; sa largeur, dans sa région antérieure, est égale à $0,014 \mathrm{~mm}$. La pointe recourbée mesure $0,038 \mathrm{~mm}$ de longueur pour un diamètre à sa base de $0,007 \mathrm{~mm}$. Des muscles puissants viennent s'insérer à la base de ces crochets, principalement à l'extrémité antérieure de la garde.

Notons que la forme de ces crochets est à très peu de chose près superposable à celle des hamulis chez les espèces du genre Polystoma. 
Les crochets de la paire que nous qualifions d'externe ont des dimensions comparables à celles des précédents, mais, chez eux, l'insertion de la garde sur le manche ne se fait plus suivant un angle de $45^{\circ}$ : manche et garde sont rapprochés, sub-parallèles, de largeur un peu différente $(0,012 \mathrm{~mm}$ pour la garde ; $0,008 \mathrm{~mm}$ pour le manche), et l'on n'observe plus entre le manche et la garde qu'une simple encoche, presque virtuelle. La pointe de ces crochets est légèrement plus petite que celle des crochets de la paire interne.

b) Pièces intermédiaires : Il existe de manière constante, au voisinage des crochets précédents, une série de pièces de nature apparemment identique à la leur, mais de forme assez variable; on peut distinguer une paire de pièces grossièrement ovoïdes, mesurant 0,045 sur $0,015 \mathrm{~mm}$, très près desquelles prennent place deux pièces de forme semblable, mais plus petites $(0,014$ sur $0,008 \mathrm{~mm})$; il peut exister aussi quelques corpuscules supplémentaires de forme et de position très irrégulières.

c) Crochets larvaires : Ils sont en position marginale tout le tour du hapteur, sauf la paire morphologiquement la plus postérieure qui est superposée aux grands crochets (fig. 2). Au total, il existe seize crochets larvaires, nombre qui a été vérifié à la fois sur le vivant et sur les préparations in toto. Ils mesurent $0,015 \mathrm{~mm}$ de longueur. Ils sont pourvus d'une pointe fortement recourbée et d'une garde courte, insérée aux deux tiers de la longueur du crochet.

\section{APPAREIL DIGESTIF :}

Bouche sub-terminale ventrale, s'ouvrant entre les deux ventouses antérieures; elle est immédiatement en relation avec un pharynx important, organe sphérique mesurant $0,30 \mathrm{~mm}$ de diamètre en moyenne. Au pharynx font suite sans transition les deux branches de l'appareil digestif qui se rejoignent en arrière du corps (appareil digestif « en anneau »); elles ont un diamètre régulier $(0,05 \mathrm{~mm})$ et ne présentent pas de diverticules. Il existe des glandes digestives latérales qui débouchent juste en arrière du pharynx.

\section{TACHES OCULAIRES :}

La région antérieure, jusqu'au niveau du bord postérieur du pharynx, est couverte d'une multitude de petites taches oculaires rouge sombre, chacune de ces taches étant constituée d'un à dix corpuscules élémentaires. Ces corpuscules sont soit superficiels, soit profonds ; leur distribution paraît quelconque, bien que la face dorsale en soit plus richement pourvue. Ces taches existent chez tous nos exemplaires.

\section{APPAREIl GÉNITAL MALE :}

Testicule unique, volumineux, de forme irrégulière, mais non lobée, occupant la moitié postérieure du corps. Le canal déférent part de sa région antérieure, chemine dans le côté gauche du corps et va s'ouvrir (fig. 3 A) à la base de deux épines génitales, entre lesquelles se trouve un cirre sclérifié. Les épines génitales mesurent 0,028 sur $0,004 \mathrm{~mm}$; elles se terminent en une pointe légèrement recourbée ; cette pointe est dirigée vers l'arrière sur les préparations in toto. 
APPAREIL gÉNITAL FEMElle :

Ovaire situé en avant du testicule, dans la moitié droite du corps, ventralement par rapport au tube digestif. Il est de forme allongée, sa partie postérieure étant trapue, tandis qu'il s'effile antérieurement. Il renferme des ovocytes nombreux, d'autant plus
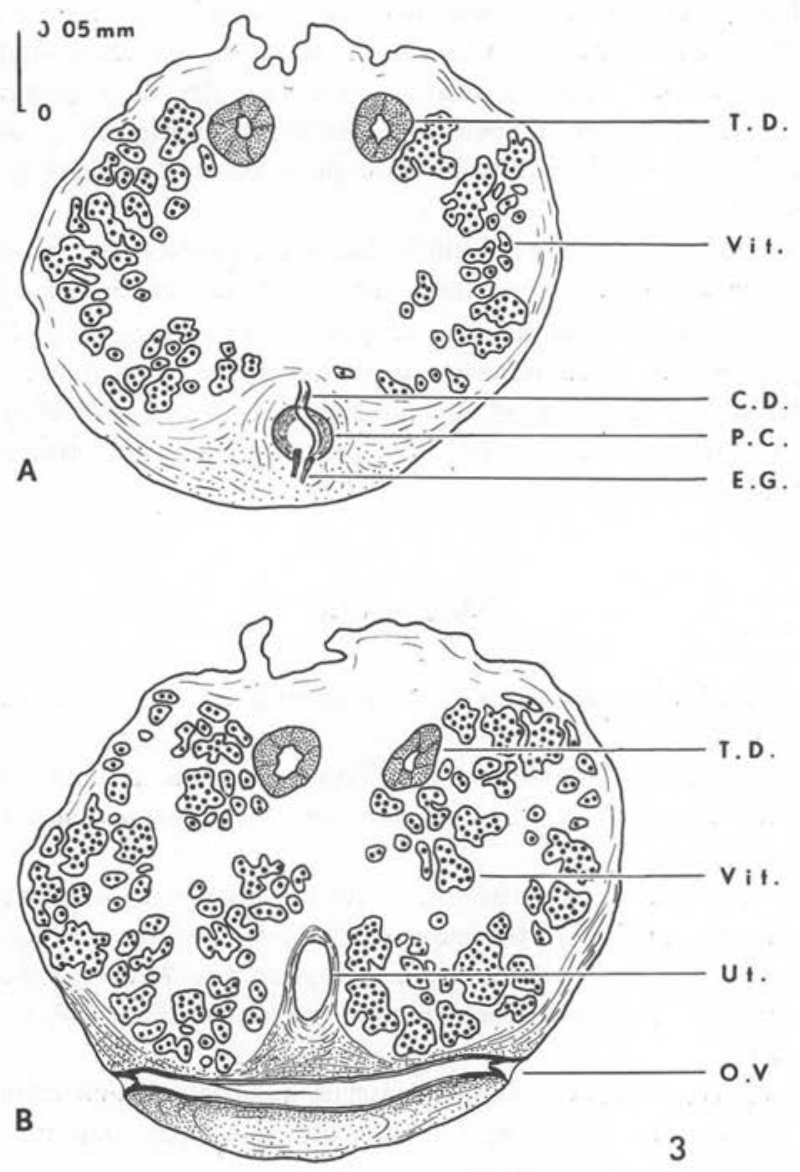

Fig. 3. - E. knoepffleri; coupes transversales: A) au niveau de la poche du cirre. B) au niveau des vagins. C.D. = canal déférent. E.G. = épine génitale. O.V. = ouverture vaginale. P.C. $=$ poche du cirre. T.D. = tube digestif. Ut. $=$ utérus. Vit. = vitellogènes

volumineux qu'on se rapproche de l'oviducte. Celui-ci se courbe vers l'arrière, puis forme un deuxième coude après lequel il se dirige vers l'avant. Il reçoit un canal vaginal impair, puis un vitelloducte impair médian. 
Le canal vaginal impair résulte de la fusion de deux vagins latéraux qui cheminent transversalement depuis les ouvertures vaginales. Celles-ci sont situées ventralement, à droite et à gauche du corps, à $0,60 \mathrm{~mm}$ environ de l'extrémité antérieure. Elles présentent chacune, un peu à l'intérieur du corps, un système de fermeture, en forme de valve circulaire (fig. $3 \mathrm{~B}$ ), destiné peut-être à empêcher le reflux éventuel des spermatozoïdes. Il ne nous semble pas qu'un dispositif identique soit connu chez les Monogènes.

Le vitelloducte impair médian provient de la fusion de deux vitelloductes transverses, chacun de ceux-ci étant en relation avec l'un des deux groupes latéraux de vitellogènes ; ceux-ci s'étendent à l'extérieur des branches digestives, depuis la région du pharynx jusqu'au niveau du bord antérieur du hapteur. Les deux groupes se rejoignent ou non en arrière.

Il faut noter que les vagins et les vitelloductes transverses sont presque superposés. Les structures ci-dessus ont été confirmées par l'étude de coupes transversales sériées.

Après le carrefour du vitelloducte médian, l'utérus succède à l'oviducte et va déboucher au pore génital après un court trajet, au même point que le canal déférent. L'utérus contient souvent un œuf, et un seulement, de forme très allongée, sub-cylindrique, pourvu à l'une de ses extrémités d'un petit ornement en forme de vrille. L'œuf mesure 0,17 sur $0,07 \mathrm{~mm}$ (fig. 1).

\section{Discussion}

Ce parasite, dont nous soulignons l'habitat interne, est, par ses caractères généraux, un Monogenea Monopisthocotylea.

Sa position systématique et son écologie l'opposent donc aux Sphyranuridae (seuls Monogènes connus chez les Urodèles) qui sont des Polyopisthocotylea et vivent sur la peau ou les branchies.

Presque tous les Monopisthocotylea parasitent d'ailleurs des Poissons. Gyrdicotylus gallieni Vercammen-Grandjean 1960, parasite intestinal de Xenopus laevis victorianus, Amphibien Anoure du Congo. et Iagotrema uruguayensis Mañé-Garzón et Gil 1962, de la vessie urinaire de Hydromedusa tectifera, Chélonien d'eau douce d'Amérique du Sud, font exception.

A notre connaissance, aucun parasite interne appartenant aux Monogenea n'a été décrit chez les Amphibiens Urodèles. L'espèce que nous décrivons présente donc une écologie nouvelle pour les Monogenea.

Iagotrema uruguayensis présente certaines analogies morphologiques et anatomiques avec notre espèce, mais il s'en distingue, outre plusieurs différences portant sur la forme des organes, des crochets et de l'œuf, par l'absence de vagin et le hapteur à quatorze crochets larvaires seulement.

Le parasite de l'Euprocte de Corse ne paraît entrer dans aucun genre connu. Nous proposons la création du genre Euzetrema, en hommage à M. le Professeur Louis Euzet. Notre espèce portera le nom de M. Louis-Philippe Knoepffler, qui nous a procuré la première récolte d'Euproctus montanus. 
Vu l'importance que prend la morphologie de l'oncomiracidium dans la systématique des Monogènes, nous attendons d'avoir étudié la larve d'Euzetrema pour nous prononcer sur son attribution familiale.

(Laboratoire de Zoologie et Biologie animale, Collège Scientifique Universitaire de Perpignan) (Pyr.-Orient.)

\section{Bibliographie}

BYchowsKy (B. E.), 1957. - Systématique et phylogénie des Trématodes Monogènes (en russe). Pub. Lab. Zool. Acad. Sc. U.R.S.S., Léningrad, p. 1-509, fig. 1-315.

Goux (L.), 1953. - Contribution à l'étude biogéographique, écologique et biologique de l'Euprocte de Corse. Vie et Milieu, 6 (1), p. 1-36.

Goux (L.), 1955. - Nouvelles observations sur la biogéographie, l'écologie et la biologie de l'Euprocte de Corse, Euproctus montanus (Savi) (Salamandridae). Vie et Milieu, 6 (3), p. 299-317, fig. 1-5.

Mañé-Garzón (F.) et GIL (O.), 1962. - Trematodos de las Tortugas del Uruguay, VI. Iagotrema uruguayensis $\mathrm{n}$. gen., n. sp., Monogenea Monopisthocotylea, de la vesiga urinaria de Hydromedusa tectifera (Cope). Com. zool. Mus. Hist. Nat. Montevideo, 7 (98), p. 1-7, 4 pl.

Sproston (N. G.), 1946. - A synopsis of the Monogenetic Trematodes. Trans. Zool. Soc. Lond., 25, p. 185-600, fig. 1-118.

Vercammen-Grandjean (T. H.), 1960, - Les Trématodes du lac Kivu-Sud (Vermes). Ann. K. Mus. Midden-Africa, Tervuren, n.s. 5, p. 1-171.

YAMAgUti (S.), 1963. - Systema helminthum. IV. Monogenea and Aspidocotylea. London, pp. 1-699, fig. 1-898. 